\title{
Municipal Solid Waste Management under Uncertainty: An Interval-Fuzzy Two-Stage Stochastic Programming Approach
}

\author{
Y. P. $\mathrm{Li}^{1}$, G. H. Huang ${ }^{2, *}$, and H. N. Xiao ${ }^{3}$ \\ ${ }^{I}$ College of Urban and Environmental Sciences, Peking University, Beijing 100871, China \\ ${ }^{2}$ Environmental Systems Engineering Program, Faculty of Engineering, University of Regina, Regina, SK S4S 0A2, Canada \\ ${ }^{3}$ Department of Chemical Engineering, University of New Brunswick, 15 Dineen Drive, Fredericton, NB E3B 5A3, Canada
}

Received 15 December 2007; revised 25 March 2008; accepted 28 May 2008; published online 1 December 2008

\begin{abstract}
In this study, an interval-fuzzy two-stage stochastic linear programming (IFTP) method is developed for planning waste-management systems under uncertainty. In the IFTP, approaches of two-stage stochastic programming, interval-parameter programming, and fuzzy linear programming are integrated into a general optimization framework to effectively tackle uncertainties described in terms of probability density functions, fuzzy membership functions and discrete intervals. The IFTP method can incorporate pre-regulated waste management policies directly into its optimization process, and be used for analyzing various policy scenarios that are associated with different levels of economic penalties when the promised policy targets are violated. It can also help quantify the satisfaction degrees of the system objective and constraints under uncertainty, as defined in the obtained solutions. The IFTP model can be transformed into two deterministic submodels based on an interactive algorithm. Interval solutions, which are stable in the given decision space with varying levels of system-failure risk, can then be obtained by solving the two submodels sequentially. Then, the developed method is applied to a case study of waste allocation within a municipal solid waste management system. The results indicate that reasonable solutions have been generated. They can be used to generate decision alternatives and help MSW managers to identify desired policies under various environmental, economic, and system-reliability conditions
\end{abstract}

Keywords: decision-making, environment, fuzzy, interval optimization, solid waste, two-stage, uncertainty

\section{Introduction}

The majority of the previous methods dealing with such uncertainties include fuzzy mathematical programming (FMP), stochastic mathematical programming (SMP), and interval mathematical programming (IMP) (Baetz, 1990; Chang and Wang, 1997; Chang et al., 1997; Huang et al., 1992, 1993, 2001; Chanas and Zielinski, 2000; Li and Huang, 2006a, b). FMP considers uncertainties as fuzzy sets, and is effective in reflecting ambiguity and vagueness in resource availabilities. In comparison, IMP method can deal with uncertainties expressed as intervals in objective-function coefficients and constraints' left- and right-hand sides (Huang et al., 1992). Consequently, combining advantages of FMP and IMP, Huang et al. (1993) proposed an interval-fuzzy programming method and applied it to MSW management systems to tackle uncertainties presented in terms of fuzzy and interval forms. Recently, Huang et al. (2001) developed an integrated fuzzy-stochastic linear programming method and applied it to MSW management, in which chance-constrained programming and

\footnotetext{
* Corresponding author. Tel.: +1 306 5854095; fax: +1 3065854855.

E-mail address: gordon.huang@uregina.ca (G. H. Huang).
}

ISSN: 1726-2135 print/1684-8799 online

(C) 2008 ISEIS All rights reserved. doi:10.3808/jei.200800128 fuzzy linear programming were incorporated within a general interval-parameter mixed integer linear programming framework. In fact, although chance-constrained and fuzzy programming methods can effectively reflect probabilistic or possibilistic distributions of a linear model's right-hand sides, they cannot handle independent uncertainties of its left-hand sides and cost coefficients. Moreover, they are lack of linkage to economic consequences of violated policies predefined by authorities through taking recourse actions in order to correct any infeasibilities.

Two-stage stochastic programming (TSP) is an effective tool for handling optimization problems where an analysis of policy scenarios is desired and the right-hand-side coefficients are random with known probability distributions. The fundamental idea behind the stochastic programming is the concept of recourse, which is the ability to take corrective actions after a random event has taken place. In the TSP, a decision is first undertaken before values of random variables are known; then, after the random events have happened and their values are known, a second-stage decision can be made in order to minimize "penalties" that may appear due to any infeasibility (Loucks et al., 1981; Birge and Louveaux, 1988, 1997). The initial action is called the first-stage decision, and the corrective one is named the second-stage decision. The TSP methodologies were applied to a variety of problems over the past 
decades (Schultz et al., 1996; Ruszczynski and Swietanowski, 1997; Ferrero et al., 1998; Huang and Loucks, 2000; Seifi and Hipel, 2001; Luo et al., 2003; Maqsood and Huang, 2003; Li et al., 2006, 2007). For example, Ferrero et al. (1998) examined hydrothermal scheduling of multi-reservoir systems using a two-stage algorithm; Huang and Loucks (2000) proposed an inexact two-stage stochastic programming (ITSP) method for water resources management, which could tackle uncertainties expressed as both probability distributions and intervals and account for economic penalties. However, few previous studies were reported on the development of TSP methods for solid waste management. Maqsood and Huang (2003) firstly explored a two-stage interval-stochastic programming (TISP) method for the planning of solid waste management. The TISP can deal with uncertainties expressed as probability density functions and discrete intervals; however, it can hardly reflect the system's dynamic feature (e.g. planning for capacity expansions) and, at the same time, has difficulties when the model's right-hand-side parameters have large intervals. Li et al. (2006) proposed an interval-parameter two-stage stochastic integer programming method for planning solid-waste management systems; this method can facilitate dynamic analysis for decisions of capacity-expansion planning within a multiperiod context and under stochastic conditions. However, it was incapable of tackling uncertainties in the constraints' right-hand sides presented as fuzzy sets. In fact, in many realworld problems, results produced by optimization techniques can be rendered highly questionable if the modeling inputs cannot be expressed with precision. Quality of the available information is generally poor, and uncertainties may be presented as random variables, intervals and/or fuzzy sets (Yeomans and Huang, 2003; Li and Huang, 2006a). Therefore, a robust reflection of the variety of uncertainties is desired.

Thus, the objective of this study is to develop an intervalfuzzy two-stage stochastic linear programming (IFTP) method for planning waste-management systems. The method can directly deal with uncertainties expressed as fuzzy membership functions, probability density functions and discrete intervals. Moreover, it can support the analysis of various policy scenarios that are associated with different levels of economic penalties when the promised targets are violated. The proposed method will then be applied to a case study of MSW management to demonstrate its applicability. The results can help quantify the relationships between system cost and degree of satisfaction, and thus generate desired decision alternatives. This will allow in-depth analyses of tradeoffs between environmental and economic objectives as well as those between system cost and decision-makers' satisfaction degree.

\section{Model Development}

\subsection{Interval-Fuzzy Linear Programming}

Consider an interval-parameter programming (IPP) model as follows (Huang et al., 1992):

$\operatorname{Min} f^{ \pm}=C^{ \pm} X^{ \pm}$

subject to:
$A^{ \pm} X^{ \pm} \leq B^{ \pm}$

$X^{ \pm} \geq 0$

where $A^{ \pm} \in\left\{R^{ \pm}\right\}^{\mathrm{m} \times \mathrm{n}}, B^{ \pm} \in\left\{R^{ \pm}\right\}^{\mathrm{mx} 1}, C^{ \pm} \in\left\{R^{ \pm}\right\}^{1 \times \mathrm{n}}, X^{ \pm} \in\left\{R^{ \pm}\right\}^{\mathrm{n} \times 1}$, $R^{ \pm}$denotes a set of interval numbers, and $f^{ \pm}$refers to a linear objective function. This IPP model can be transformed into two deterministic submodels corresponding to upper and lower bounds of the objective function value (Huang et al., 1992). By solving the two submodels, interval solutions can be obtained. Furthermore, when the system's goal and constraints are fuzzy, an interval-fuzzy linear programming (IFLP) model can be formulated through incorporating the concept of fuzzy programming within the IPP framework (Huang et al., 1993):

$\operatorname{Max} \lambda$

subject to:

$C^{ \pm} X^{ \pm} \leq f^{+}-\lambda\left(f^{+}-f^{-}\right)$

$A^{ \pm} X^{ \pm} \leq B^{+}-\lambda\left(B^{+}-B^{-}\right)$

$X^{ \pm} \geq 0$

$0 \leq \lambda \leq 1$

where $f^{-}$and $f^{+}$are lower and upper bounds of the objective's aspiration level, respectively. Based on the principle of fuzzy flexible programming (Zimmermann, 1985), $\lambda$ is a control variable corresponding to the degree (membership grade) of satisfaction for a fuzzy decision. Specifically, the flexibility in the constraints and fuzziness in the objective (which are represented by fuzzy sets and denoted as "fuzzy constraints" and a "fuzzy goal”, respectively) are expressed as membership grades $(\lambda)$ corresponding to the degrees of overall satisfaction for the constraints/objective (Zimmermann, 1985; Huang et al., 1993).

\subsection{Two-Stage Stochastic Programming}

When uncertainties in the model's right-hand-side values are expressed as probability density functions while decisions need to be made periodically over time, the study problem can be formulated as a two-stage stochastic programming (TSP) model. In the TSP, the decision variables are divided into two subsets: those that must be determined before the realizations of random variables are known, and those (recourse variables) that will be determined after the realized values of the random variables are available. This implies that a second-stage decision is used to minimize "penalties" that may appear due to any infeasibility. Assume that B of Model (1) with a minimized objective and equality constraints is not precisely known and only its distribution, with finite mean $E(B)$, is given. We can then assume that there exists a penalty for any difference (a random variable) between $\mathrm{AX}$ and $\mathrm{B}$. The problem can thus 
become to choose $\mathrm{X}$ which minimizes the sum of the $\mathrm{CX}$ and the mean of the penalties (Dantzig, 1983). A standard formulation for a TSP model is provided as follows (Birge and Louveaux, 1997):

$z=\min C^{T} X+E_{\omega \in \Omega}[Q(X, \omega)]$

s.t. $x \in X$

with

$Q(x, \omega)=\min f(\omega)^{T} y$

s.t. $D(\omega) y \geq h(\omega)+T(\omega) x$

$y \in Y$

where $X \subseteq R^{n 1}$, с $\in R^{n 1}, Y \subseteq R^{n 2}$, and $\omega$ is a random variable from probability space $(\Omega, F, P)$ with $\Omega \subseteq R^{k}, f: \Omega \rightarrow R^{n 2}, h$ : $\Omega \rightarrow R^{m 2}, D: \Omega \rightarrow R^{m 2 \times n 2}$, and $T: \Omega \rightarrow R^{m 2 \times n 1}$. Problem (3a) with variables $x$ constitutes the first-stage decision which needs to be made prior to the realization of uncertain parameters $\omega$, while problem (3b) with variables $y$ constitutes the second-stage decision. For given values of the first-stage variables $(x)$, the second-stage problem can be decomposed into independent linear sub-problems, with one sub-problem for each realization of the uncertain parameters.

\subsection{Interval-Fuzzy Two-Stage Stochastic Programming}

Consider a waste-management system wherein a manager is responsible for allocating waste flows from two cities to one landfill and one incinerator over several time periods. The objective of the study problem is to minimize the system cost with the optimal waste flow patterns. As waste-generation amounts from the cities are uncertain (expressed as probability density functions) while decisions need to be made periodically over time, the study problem can be formulated as a twostage stochastic programming (TSP) model. A decision of target waste-flow allocation made at the beginning is named the first-stage decision, and the recourse decision is called the second-stage decision. The first-stage decision has to be made before further information is revealed, whereas the secondstage one is to adapt to the previous decision based on the further information. Based on the local waste management policies, a projected allowable waste-flow level from each city is pre-regulated. If this level is not exceeded, it will result in a regular (normal) cost to the system; however, if this allowance is exceeded, it will mean a surplus flow associated with economic penalties and/or expansion costs. The penalties will be disposed of at a premium expressed in terms of raised transportation and operation costs; facility expansions will help increase the allowable flow levels and reduce the penalties. Thus, the total waste flow will be the sum of both fixed target and probabilistic surplus flows.

In fact, in real-world MSW management problems, the quality of available information is generally poor, being often presented as vague values and/or discrete intervals. For example, the uncertainties in waste transportation costs, facility operation costs and revenues, and waste-generation rates may be described as intervals; at the same time, the lower and upper bounds of these interval parameters may also be fuzzy in nature, leading to dual uncertainties. Thus, the IFLP and TSP methods will be integrated within a general optimization framework to deal with such uncertainties. This leads to an intervalfuzzy two-stage stochastic programming (IFTP) model as follows:

$\operatorname{Max} \lambda$

subject to:

$\sum_{i=1}^{2} \sum_{j=1}^{2} \sum_{k=1}^{3} L_{k} X_{i j k}^{ \pm}\left(T R_{i j k}^{ \pm}+O P_{i k}^{ \pm}\right)+\sum_{j=1}^{2} \sum_{k=1}^{3} L_{k} X_{2 j k}^{ \pm} F E_{2}^{ \pm}\left(F T_{2 k}^{ \pm}+O P_{1 k}^{ \pm}\right)$
$+\sum_{i=1}^{2} \sum_{j=1}^{2} \sum_{k=1}^{3} L_{k} E\left[M_{i j k}^{(w)}\right]\left(D R_{i j k}^{ \pm}+D P_{i k}^{ \pm}\right)+\sum_{j=1}^{2} \sum_{k=1}^{2} L_{k} E\left[M_{i j k}^{(w)}\right] F E_{2}^{ \pm}$.
$\left(D T_{2 k}^{ \pm}+D P_{1 k}^{ \pm}\right)-\sum_{j=1}^{2} \sum_{k=1}^{3} L_{k} X_{i j k}^{ \pm} R E_{2 k}^{ \pm}-\sum_{j=1}^{2} \sum_{k=1}^{3} L_{k} E\left[M_{i j k}^{(w)}\right] R M_{2 k}^{ \pm}$
$\leq f^{-}+(1-\lambda)\left(f^{+}-f^{-}\right)$

[system objective constraint]

$\sum_{j=1}^{2} \sum_{k=1}^{3} L_{k}\left[\left(X_{1 j k}^{ \pm}+M_{1 j k}^{(w)}\right)+F E_{2}^{ \pm}\left(X_{2 j k}^{ \pm}+M_{2 j k}^{(w)}\right)\right] \leq L \underline{C}+(1-\lambda)(L \bar{C}$

$-L \underline{C})$

[landfill-capacity constraint]

$\sum_{j=1}^{2}\left(X_{2 j k}^{ \pm}+M_{2 j k}^{(w)}\right) \leq T \underline{C}+(1-\lambda)(T \bar{C}-T \underline{C})$

[incinerator-capacity constraint]

$\sum_{i=1}^{2}\left(X_{i j k}^{ \pm}+M_{i j k}^{(w)}\right) \geq \underset{\sim j k}{W_{j k}^{-}}+\lambda \Delta \underset{\sim j k}{W_{j k}^{ \pm}}, \forall j, k$

[waste-disposal demand constraint]

$\sum_{j=1}^{2}\left(X_{2 j k}^{ \pm}+M_{2 j k}^{(w)}\right) \geq D G_{2 k}^{ \pm} \sum_{j=1}^{2}\left(W_{j k}^{-}+\lambda \Delta{\underset{\sim}{j k}}_{j k}^{ \pm}\right), \forall k$

[diversion-rate constraint of waste flows to incinerator]

$X_{i j k}^{ \pm} \geq M_{i j k}^{(w)} \geq 0, \forall i, j, k$

$0 \leq \lambda \leq 1$

[non-negativity and technical constraints]

where:

$i=$ type of waste management facility, where $i=1$ for landfill, and $i=2$ for incinerator;

$j$ = name of city, $j=1,2$;

$k$ = planning period, $k=1,2,3$;

$L_{k}=$ length of period $k$ (day); 
$D G_{2 k}^{ \pm}=$regulated diversion rate of waste flow to the incinerator in period $k(\%)$;

$D P_{i k}^{ \pm}=$operating cost of facility i for excess waste flow during period $\mathrm{k}$ (the second-stage cost parameter) (\$/t), where $D P_{i k}^{ \pm} \geq O P_{i k}^{ \pm}$

$D R_{i j k}^{ \pm}=$transportation cost for excess waste flow from city $j$ to facility $i$ during period $k$ (the second-stage cost parameter) (\$/t), where $D R_{i j k}^{ \pm} \geq T R_{i j k}^{ \pm}$;

$D T_{i k}^{ \pm}=$transportation cost of excess waste residue from the incinerator to the landfill during period $k$ (the second-stage cost parameter) (\$/t), where $D T_{i k}^{ \pm} \geq F T_{i k}^{ \pm}$;

$F E_{2}^{ \pm}=$residue flow rate from the incinerator to the landfill (\% of incoming mass to facility $i$ );

$F T_{2 k}^{ \pm}=$transportation cost for allowable residue flow from the incinerator to the landfill during period $k(\$ / t)$;

$L \underline{C}=$ lower bound of the landfill capacity (tonne);

$L \bar{C}=$ upper-bound of the landfill capacity (tonne);

$M_{i j k}^{(w)}=$ amount by which the allowable waste flow level $\left(X_{i j k}\right)$ is exceeded when the waste generation rate in city $j$ during period $k$ is $W_{j k}(\mathrm{t} / \mathrm{d})$ (the second-stage decision variable);

$O P_{i k}^{ \pm}=$operating cost of facility i for allowable waste flow during period $k(\$ / \mathrm{t})$;

$R E_{2 k}^{ \pm}=$revenue from allowable waste flow treated by the incinerator during period $k$ (the first-stage cost parameter) $(\$ / \mathrm{t})$;

$R M_{2 k}^{ \pm}=$revenue from excess flow treated by the incinerator during period $k$ (the second-stage cost parameter) (\$/t);

$T \underline{C}=$ lower-bound of the incinerator capacity (t/d);

$T \bar{C}=$ upper-bound of the incinerator capacity (t/d);

$T R_{i j k}^{ \pm}=$transportation cost for allowable waste flow from city $j$ to facility $i$ during period $k$ (the first-stage cost parameter) $(\$ / t)$;

$W_{j k}=$ random variable of waste-generation rate in city $j$ during period $k(\mathrm{t} / \mathrm{d})$;

$X_{i j k}^{ \pm}=$allowable waste flow from city $j$ to facility $i$ during period $k$ (the first-stage variable) (t/d);

where $f^{-}$and $f^{+}$are the lower and upper bounds of the objective's aspiration level as designated by decision makers, respectively; $\lambda$ is control variable and $M_{i j k}^{(w)}$ are decision variables. The $\lambda$ value corresponds to the degree of satisfaction for the fuzzy objective and/or constraints, which ranges between 0 and 1 . A value closer to 1 would correspond to a solution with a high possibility of satisfying the constraints/ objective under advantageous conditions; conversely, a value near 0 would be related to a solution that has a low possibility of satisfying the constraints/objective under demanding conditions. In model (4), $\Delta W_{j k}^{ \pm}=W_{\sim j k}^{+}-W_{j k}^{-}=\left[\underline{W}_{j k}^{+}, \bar{W}_{j k}^{+}\right]-\left[\underline{W}_{j k}^{-}, \bar{W}_{j k}^{-}\right]$ $=\left[\underline{W}_{j k}^{+}-\bar{W}_{j k}^{-}, \bar{W}_{j k}^{+}-\underline{W}_{j k}^{-}\right]$, where $\underline{W}_{j k}^{-}$is the lower boundary of lower interval waste-generation rate, and $\bar{W}_{j k}^{-}$is the upper boundary of lower interval waste-generation rate, and $\underline{W}_{j k}^{+}$is the lower boundary of upper interval waste-generation rate, and $\bar{W}_{j k}^{+}$is the upper boundary of upper interval waste-generation rate, respectively.
Model (4) is generally nonlinear, and the set of feasible constraints is convex only for some particular distributions. However, the IFTP problem can be equivalently formulated as a linear model (Maqsood and Huang, 2003). Let each $W_{j k}$ take value $w_{j k h}$ with probability $p_{j h}$ (for $h=1,2, \ldots, \mathrm{s}$ ), where $h$ is denoted as the level of waste-generation rate in city $j$. Consequently, the above nonlinear model can be converted into a linear one (IFTP) as follows:

$\operatorname{Max} \lambda$

subject to:

$$
\begin{aligned}
& \sum_{i=1}^{2} \sum_{j=1}^{2} \sum_{k=1}^{3} L_{k} X_{i j k}^{ \pm}\left(T R_{i j k}^{ \pm}+O P_{i k}^{ \pm}\right)+\sum_{j=1}^{2} \sum_{k=1}^{3} L_{k} X_{2 j k}^{ \pm} F E_{2}^{ \pm}\left(F T_{2 k}^{ \pm}+O P_{1 k}^{ \pm}\right) \\
& +\sum_{i=1}^{2} \sum_{j=1}^{2} \sum_{k=1}^{3} \sum_{h=1}^{s} L_{k} p_{j h} M_{i j k h}^{ \pm}\left(D R_{i j k}^{ \pm}+D P_{i k}^{ \pm}\right)+\sum_{j=1}^{2} \sum_{k=1}^{2} \sum_{h=1}^{s} L_{k} p_{j h} M_{i j k h}^{ \pm} F E_{2}^{ \pm} . \\
& \left(D T_{2 k}^{ \pm}+D P_{1 k}^{ \pm}\right)-\sum_{j=1}^{2} \sum_{k=1}^{3} L_{k} X_{i j k}^{ \pm} R E_{2 k}^{ \pm}-\sum_{j=1}^{2} \sum_{k=1}^{3} \sum_{h=1}^{s} L_{k} p_{j h} M_{i j k h}^{ \pm} R M_{2 k}^{ \pm} \\
& \leq f^{-}+(1-\lambda)\left(f^{+}-f^{-}\right)
\end{aligned}
$$

$\sum_{j=1}^{2} \sum_{k=1}^{3} L_{k}\left[\left(X_{1 j k}^{ \pm}+M_{1 j k h}^{ \pm}\right)+F E_{2}^{ \pm}\left(X_{2 j k}^{ \pm}+M_{2 j k}^{ \pm}\right)\right] \leq L \underline{C}+(1-\lambda)(L \bar{C}$

$-L \underline{C}), \forall h$

$\sum_{j=1}^{2}\left(X_{2 j k}^{ \pm}+M_{2 j k h}^{ \pm}\right) \leq T \underline{C}+(1-\lambda)(T \bar{C}-T \underline{C}), \quad \forall h$

$\sum_{i=1}^{2}\left(X_{i j k}^{ \pm}+M_{i j k h}^{ \pm}\right) \geq{\underset{\sim}{w j k h}}_{-}^{-}+\lambda \Delta \underset{\sim}{w_{j k h}^{ \pm}}, \forall j, k, h$

$\sum_{j=1}^{2}\left(X_{2 j k}^{ \pm}+M_{2 j k h}^{ \pm}\right) \geq D G_{2 k}^{ \pm} \sum_{j=1}^{2}\left({\underset{\sim}{j k h}}_{-}^{-}+\lambda \Delta{\underset{\sim}{j k h}}_{j}^{ \pm}\right), \quad \forall k, h$

$X_{i j k}^{ \pm} \geq M_{i j k h}^{ \pm} \geq 0, \quad \forall i, j, k, h$

$0 \leq \lambda \leq 1$

\subsection{Solution Method for the IFTP}

The IFTP model can deal with uncertainties described as not only intervals but also probability distributions and membership functions. When $X_{i j k}^{ \pm}$are known as deterministic values, the model can be transformed into two deterministic submodels that correspond to the lower and upper bounds of the objective value through a two-step solution method (Huang et al., 1993). Since the objective is to minimize the system cost, submodel with $\lambda$ corresponding to $f^{-}$(i.e. the most desirable objective value) can be first formulated as follows:

$\operatorname{Max} \lambda$

subject to: 
$\sum_{i=1}^{2} \sum_{j=1}^{2} \sum_{k=1}^{3} L_{k} X_{i j k}^{-}\left(T R_{i j k}^{-}+O P_{i k}^{-}\right)+\sum_{j=1}^{2} \sum_{k=1}^{3} L_{k} X_{2 j k}^{-} F E_{2}^{-}\left(F T_{2 k}^{-}+O P_{1 k}^{-}\right)$

$+\sum_{i=1}^{2} \sum_{j=1}^{2} \sum_{k=1}^{3} \sum_{h=1}^{s} L_{k} p_{j h} M_{i j k h}^{-}\left(D R_{i j k}^{-}+D P_{i k}^{-}\right)+\sum_{j=1}^{2} \sum_{k=1}^{2} \sum_{h=1}^{s} L_{k} p_{j h} M_{i j k h}^{-} F E_{2}^{-}$.

$\left(D T_{2 k}^{-}+D P_{1 k}^{-}\right)-\sum_{j=1}^{2} \sum_{k=1}^{3} L_{k} X_{i j k}^{-} R E_{2 k}^{+}-\sum_{j=1}^{2} \sum_{k=1}^{3} \sum_{h=1}^{s} L_{k} p_{j h} M_{i j k h}^{-} R M_{2 k}^{+}$

$\leq f^{-}+(1-\lambda)\left(f^{+}-f^{-}\right)$

$\sum_{j=1}^{2} \sum_{k=1}^{3} L_{k}\left[\left(X_{1 j k}^{-}+M_{1 j k h}^{-}\right)+F E_{2}^{-}\left(X_{2 j k}^{-}+M_{2 j k}^{-}\right)\right] \leq L \underline{C}+(1-\lambda)(L \bar{C}$

$-L \underline{C}), \forall h$

$\sum_{j=1}^{2}\left(X_{2 j k}^{-}+M_{2 j k h}^{-}\right) \leq T \underline{C}+(1-\lambda)(T \bar{C}-T \underline{C}), \quad \forall h$

$\sum_{i=1}^{2}\left(X_{i j k}^{-}+M_{i j k h}^{-}\right) \geq \underline{w}_{j k h}^{-}+\lambda\left(\underline{w}_{j k h}^{+}-\bar{w}_{j k h}^{-}\right), \forall j, k, h$

$\sum_{j=1}^{2}\left(X_{2 j k}^{-}+M_{2 j k h}^{-}\right) \geq D G_{2 k}^{-} \sum_{j=1}^{2}\left[\underline{w}_{j k h}^{-}+\lambda\left(\underline{w}_{j k h}^{+}-\bar{w}_{j k h}^{-}\right)\right], \forall k, h$

$X_{i j k}^{-} \geq M_{i j k h}^{-} \geq 0, \quad \forall i, j, k, h$

$0 \leq \lambda \leq 1$

where $\lambda$ and $M_{i j k h}^{-}$are decision variables. Let $\lambda_{\text {opt }}$ and $M_{i j k h \text { opt }}^{-}$be the solutions of submodel (6). In the second step, submodel (7) with $\lambda$ corresponding to $f^{+}$can then be formulated based on the solutions of submodel (6) as follows:

$\operatorname{Max} \lambda$

subject to:

$\sum_{i=1}^{2} \sum_{j=1}^{2} \sum_{k=1}^{3} L_{k} X_{i j k}^{+}\left(T R_{i j k}^{+}+O P_{i k}^{+}\right)+\sum_{j=1}^{2} \sum_{k=1}^{3} L_{k} X_{2 j k}^{+} F E_{2}^{+}\left(F T_{2 k}^{+}+O P_{1 k}^{+}\right)$

$+\sum_{i=1}^{2} \sum_{j=1}^{2} \sum_{k=1}^{3} \sum_{h=1}^{s} L_{k} p_{j h} M_{i j k h}^{+}\left(D R_{i j k}^{+}+D P_{i k}^{+}\right)+\sum_{j=1}^{2} \sum_{k=1}^{2} \sum_{h=1}^{s} L_{k} p_{j h} M_{i j k h}^{+} F E_{2}^{+}$.

$\left(D T_{2 k}^{+}+D P_{1 k}^{+}\right)-\sum_{j=1}^{2} \sum_{k=1}^{3} L_{k} X_{i j k}^{+} R E_{2 k}^{-}-\sum_{j=1}^{2} \sum_{k=1}^{3} \sum_{h=1}^{s} L_{k} p_{j h} M_{i j k h}^{+} R M_{2 k}^{-}$

$\leq f^{-}+(1-\lambda)\left(f^{+}-f^{-}\right)$

$\sum_{j=1}^{2} \sum_{k=1}^{3} L_{k}\left[\left(X_{1 j k}^{+}+M_{1 j k h}^{+}\right)+F E_{2}^{+}\left(X_{2 j k}^{+}+M_{2 j k}^{+}\right)\right] \leq L \underline{C}+(1-\lambda)(L \bar{C}$

$-L \underline{C}), \quad \forall h$

$\sum_{j=1}^{2}\left(X_{2 j k}^{+}+M_{2 j k h}^{+}\right) \leq T \underline{C}+(1-\lambda)(T \bar{C}-T \underline{C}), \quad \forall h$

$\sum_{i=1}^{2}\left(X_{i j k}^{+}+M_{i j k h}^{+}\right) \geq \bar{w}_{j k h}^{-}+\lambda\left(\bar{w}_{j k h}^{+}-\underline{w}_{j k h}^{-}\right), \forall j, k, h$
$\sum_{j=1}^{2}\left(X_{2 j k}^{+}+M_{2 j k h}^{+}\right) \geq D G_{2 k}^{+} \sum_{j=1}^{2}\left[\bar{w}_{j k h}^{-}+\lambda\left(\bar{w}_{j k h}^{+}-\underline{w}_{j k h}^{-}\right)\right], \forall k, h$

$X_{i j k}^{+} \geq M_{i j k h}^{+} \geq 0, \forall i, j, k, h$

$0 \leq \lambda \leq 1$

$M_{i j k h}^{+} \geq M_{i j k h \mathrm{opt}}^{-}$

where $\lambda$ and $M_{i j k h}^{+}$are decision variables. Let $\lambda_{\text {opt }}$ and $M_{i j k h \text { opt }}^{+}$be the solutions of submodel (7). Thus, we can obtain the general solutions as follows:

$M_{i j k h o p t}^{ \pm}=\left[M_{i j k h o p t}^{-}, M_{i j k h o p t}^{+}\right], \forall i, j, k, h$

$\lambda_{\mathrm{opt}}^{ \pm}=\left[\lambda_{\mathrm{opt}}^{-}, \lambda_{\mathrm{opt}}^{+}\right]$

$f_{\text {opt }}^{ \pm}=\left[f_{\text {opt }}^{-}, f_{\text {opt }}^{+}\right]$

Thus, the optimal waste-flow pattern including regular and excess flows from each city to each facility under varied waste-generation rates is:

$A_{i j k h o p t}^{ \pm}=X_{i j k}^{ \pm}+M_{i j k h o p t}^{ \pm}, \forall i, j, k, h$

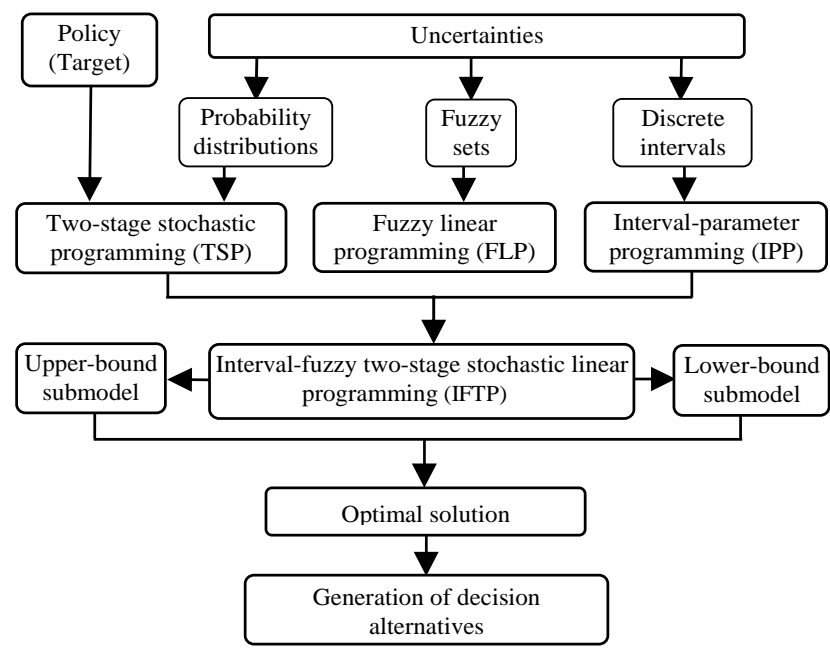

Figure 1. Framework for the IFTP method.

Figure 1 shows the framework of the IFTP model. The main advantage of the developed approach is that different policies for waste management activities can be quantitatively incorporated within the modeling framework. The IFTP model can deal with uncertainties described as intervals, probability distributions, and membership functions. The solution algorithm of the IFTP model is mainly based on an interactive algorithm. Interval solutions with an associated level of sys- 
Table 1. Waste-Generation Rates under Different Probability Levels

\begin{tabular}{|c|c|c|c|c|c|c|}
\hline \multirow{2}{*}{$\begin{array}{l}\text { Level of } \\
\text { waste-generation }\end{array}$} & \multirow{2}{*}{ Probability } & \multicolumn{5}{|c|}{ Waste-generation rate, ${\underset{\sim j k h}{ \pm}}_{j k}^{ \pm}(t / d)$} \\
\hline & & $\mathrm{k}=1$ & $\mathrm{k}=2$ & & $\mathrm{k}=3$ & \\
\hline \multicolumn{7}{|l|}{ City 1: } \\
\hline $\mathrm{h}=1(\mathrm{~L})$ & 0.2 & {$[[90,105], \quad[120,130]]$} & {$[[105,120]$,} & $[135,145]]$ & {$[[120,135]$,} & $[150,160]]$ \\
\hline $\mathrm{h}=2(\mathrm{M})$ & 0.6 & {$[[130,150], \quad[165,180]]$} & {$[[145,165]$,} & $[180,195]]$ & {$[[160,180]$,} & $[200,215]]$ \\
\hline$h=3(H)$ & 0.2 & {$[[180,205], \quad[215,230]]$} & {$[[195,220]$,} & $[235,250]]$ & {$[[215,240]$,} & $[255,270]]$ \\
\hline \multicolumn{7}{|l|}{ City 2: } \\
\hline$h=1(L)$ & 0.15 & {$[[110,125], \quad[140,150]]$} & {$[[130,145]$,} & $[160,170]]$ & {$[[155,170]$,} & $[185,195]]$ \\
\hline $\mathrm{h}=2(\mathrm{M})$ & 0.4 & {$[[150,170], \quad[185,200]]$} & {$[[170,190]$,} & $[205,220]]$ & {$[[195,215]$,} & $[230,245]]$ \\
\hline$h=3(M-H)$ & 0.3 & {$[[200,225]$,} & {$[[220,245]$,} & $[260,275]]$ & {$[[245,265]$,} & $[285,300]]$ \\
\hline $\mathrm{h}=4(\mathrm{H})$ & 0.15 & {$[[255,275]$,} & {$[[275,295]$,} & $[305,315]]$ & {$[[300,320]$,} & $[330,340]]$ \\
\hline
\end{tabular}

L: low; M: medium; M-H: medium-high; H: high.

Table 2. Regular Transportation and Operation Cost and Penalty

\begin{tabular}{|c|c|c|c|}
\hline & \multicolumn{3}{|c|}{ Time period } \\
\hline & $\mathrm{k}=1$ & $\mathrm{k}=2$ & $\mathrm{k}=3$ \\
\hline \multicolumn{4}{|l|}{ Transportation cost of allowable waste to landfill $(\$ / t)$ : } \\
\hline City 1 & {$[10.5,14.5]$} & {$[11.6,15.9]$} & {$[12.7,17.5]$} \\
\hline City 2 & {$[12.8,17.1]$} & {$[14.1,18.8]$} & {$[15.5,20.7]$} \\
\hline \multicolumn{4}{|l|}{ Transportation cost of allowable waste to incinerator $(\$ / t)$ : } \\
\hline City 1 & {$[8.8,11.7]$} & {$[9.7,12.9]$} & {$[10.7,14.2]$} \\
\hline City 2 & {$[9.6,12.8]$} & {$[10.6,14.1]$} & {$[11.7,15.5]$} \\
\hline \multicolumn{4}{|l|}{ Regular operation cost $(\$ / t)$ : } \\
\hline Landfill & {$[30,45]$} & {$[35,50]$} & {$[40,55]$} \\
\hline Incinerator & {$[50,65]$} & {$[60,80]$} & {$[70,90]$} \\
\hline Transportation cost of allowable residues (\$/t) & {$[4.7,6.3]$} & {$[5.2,6.9]$} & {$[5.7,7.6]$} \\
\hline Revenue from incinerator from allowable waste $(\$ / \mathrm{t})$ & {$[15,25]$} & {$[20,30]$} & {$[25,35]$} \\
\hline \multicolumn{4}{|l|}{ Transportation cost of excess waste to landfill $(\$ / t)$ : } \\
\hline City 1 & {$[15.8,21.8]$} & {$[17.4,23.8]$} & {$[19.0,26.3]$} \\
\hline City 2 & {$[19.2,25.6]$} & {$[21.1,28.2]$} & {$[23.2,31.0]$} \\
\hline \multicolumn{4}{|l|}{ Transportation cost of excess waste to incinerator(\$/t): } \\
\hline City 1 & {$[14.0,18.6]$} & {$[15.5,20.6]$} & {$[17.1,22.7]$} \\
\hline City 2 & {$[15.2,20.6]$} & {$[16.7,22.6]$} & {$[18.3,24.8]$} \\
\hline \multicolumn{4}{|l|}{ Operation cost of excess waste $(\$ / t)$ : } \\
\hline Landfill & {$[50,65]$} & {$[60,85]$} & {$[70,100]$} \\
\hline Incinerator & {$[100,130]$} & {$[120,160]$} & {$[140,180]$} \\
\hline Transportation cost of excess residues $(\$ / \mathrm{t})$ & {$[7.1,9.5]$} & {$[7.8,10.4]$} & {$[8.6,11.4]$} \\
\hline Revenue from incinerator from excess waste $(\$ / t)$ & {$[15,25]$} & {$[20,30]$} & {$[25,35]$} \\
\hline
\end{tabular}

tem-failure risk can be obtained through solving submodels (6) and (7) sequentially. They can be easily interpreted for generating multiple decision-making alternatives.

\section{Case Study}

Considered a case in which municipal waste flows from two cities are allocated to one landfill and one incinerator for disposal. The planning horizon is 15 years (with three 5 -year periods). The landfill has an existing capacity of 1.50 to 1.75 million tonnes, the incinerator has a capacity of 220 to 270 t/day. The incinerator generates residues of approximately $30 \%$ (on a mass basis) of its incoming waste streams, which are disposed at the landfill. In fact, the waste-generation rates may fluctuate within a range with the varied production process and major materials. Table 1 shows the waste generation rates and their associated probabilities of occurrence at the two cities. Table 2 contains transportation costs for allowable waste flows from the two cities to the landfill and the incinerator, operating costs of the landfill and incinerator, penalties for surplus waste flows, and revenues from the incinerator over the planning horizon. According to the waste management policy, an allowable waste-flow level that is a projected quota of waste flow from each city to each facility is predefined by local waste managers. Table 3 gives the allowable waste-flow levels from the two cities to landfill and incinerator, respectively. In addition, to reduce the waste flows disposed at the landfill, the region projects that the proportion of 
waste flows allocated to the incinerator will not be less than $35 \%$.

Table 3. Allowable Waste Flows from the City to the Two Facilities

\begin{tabular}{lccc}
\hline & \multicolumn{3}{c}{ Time period } \\
\cline { 2 - 4 } & $\mathrm{k}=1$ & $\mathrm{k}=2$ & $\mathrm{k}=3$ \\
\hline Allowable waste flow to landfill (t/d): & \\
City 1 & 85 & 95 & 105 \\
City 2 & 95 & 105 & 115 \\
Allowable waste flow to incinerator (t/d): & \\
City 1 & 55 & 60 & 65 \\
City 2 & 70 & 80 & 90 \\
\hline
\end{tabular}

Therefore, the problem under consideration is how to effectively allocate waste flows from the two cities to suitable waste management facilities with the minimized system cost under varied waste generation and management conditions. The decision variables represent probabilistic excess waste flows from city $j$ to facility $i$ in period $k$ under waste-generation level $h$ (denoted as $M^{ \pm} i j k$ ). The constraints involve all relationships among the decision variables and the waste generation/management conditions. Information for a variety of system components in such a waste management system is not known with certainty. Uncertainties may exist in terms of intervals, probability distributions and fuzzy membership functions; moreover, a linkage to the predefined policies as formulated by local authorities is desired. Thus, the IFTP method is considered to be a suitable approach for tackling this planning problem.

\section{Result Analysis}

Table 4 presents the solutions obtained through the IFTP method. The temporal and spatial variations of waste-generation rates may result in the varied waste-flow patterns. Each optimized waste flow is the sum of the allowable and excess flows (i.e. $A^{ \pm}{ }_{i j k h o p t}=X^{ \pm}{ }_{i j k}+M^{ \pm}{ }_{i j k h o p t}$ ) from a city to a facility un- der a given waste-generation condition. In case of excess waste, allotment to the landfill should be assigned firstly and then to incinerator due mainly to their differences in operating costs. The analysis of the modeling solutions for period 1 are provided below, while those for periods 2 and 3 can be similarly interpreted based on Table 4.

Figure 2 shows the optimized waste flows (including allowable and excess flows) from the two cities to the landfill under different waste-generation rates. For city 1, when wastegeneration rates are low, medium and high, the excess waste flows to the landfill would be $0,[4.0,20.5]$ and [33.2, 42.3] t/day (with probabilities of 20, 60 and 20\%, respectively); thus, the total waste flows from city 1 to the landfill would be 85, [89.0, 105.5] and [118.2, 127.3] t/day. For city 2, when waste-generation rates are low, medium, medium-high and high, the excess flows to the landfill would be 0 , [0.0, 15.5], [49.0, 71.6] and [81.7, 100.8] t/day (with probabilities of 15, 40,30 and $15 \%$, respectively); thus, the total waste flows from city 2 to the landfill would be 95, [95.0, 110.5], [144.0,
166.6] and [176.7, 195.8] t/day.

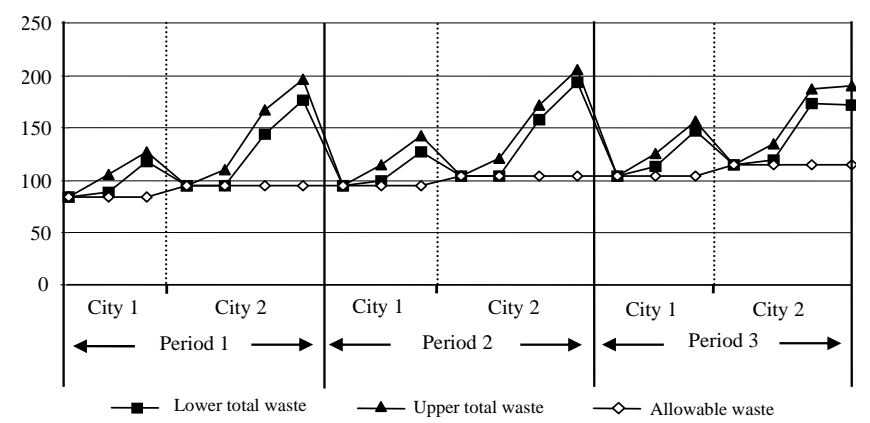

Figure 2. Optimized waste flows from the two cities to the landfill.

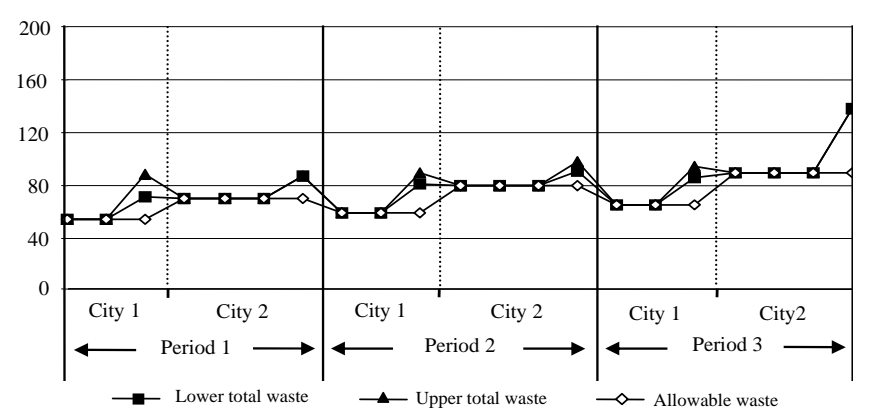

Figure 3. Optimized waste flows from the two cities to the incinerator.

Figure 3 provides the optimized waste flows (including allowable and excess flows) from the two cities to the incinerator under different waste-generation rates. For city 1, the excess flow to the incinerator would be zero when waste-generation rates are low and medium, and the total waste flows from city 1 to the incinerator would be 55 t/day; the excess flows to the incinerator would be [16.2, 33.2] t/day when waste-genera tion rate is high, and the total waste flow would be [71.2, 88.2] t/day. For city 2, there would be no excess flow to the incinerator when waste-generation rates are low, medium and medium-high, and the total waste flows from city 2 to the incinerator would be $70 \mathrm{t} / \mathrm{day}$; the excess flow to the incinerator would be 17.6 t/day when waste-generation rate is high, and the total waste flow would be $87.6 \mathrm{t} / \mathrm{day}$.

The expected system cost is $\$[115.8,191.1]$ million, with the degree of overall satisfaction ( $\lambda^{ \pm}$) being [0.21, 0.93]. The lower system cost value represents as an alternative with a lower waste generation rate, while the higher one corresponds to an alternative with a higher waste generation rate. Usually, planning with a higher system cost would guarantee that the waste management requirements and environmental regulations are met; in comparison, as the plan aims toward a lower system cost, these requirements may not be met. Therefore, in practical problems, lower decision variable values generally should be used under lower waste generation conditions since they correspond to a lower system cost; in comparison, higher decision variable values are suitable for more conservative 
Table 4. Solutions Obtained from the IFTP Model

\begin{tabular}{|c|c|c|c|c|c|c|c|c|}
\hline ijkh & Facility & City & Period & $\begin{array}{c}\text { Waste } \\
\text { generation rate }\end{array}$ & Probability (\%) & $\begin{array}{c}\text { Allowable } \\
\text { waste flow (t/d) }\end{array}$ & $\begin{array}{l}\text { Excess waste } \\
\text { flow }(\mathrm{t} / \mathrm{d})\end{array}$ & $\begin{array}{c}\text { Optimized waste } \\
\text { flow (t/d) }\end{array}$ \\
\hline 1111 & Landfill & 1 & 1 & Low & 20 & 85 & 0 & 85 \\
\hline 1112 & Landfill & 1 & 1 & Medium & 60 & 85 & {$[4.0,20.5]$} & {$[89.0,105.5]$} \\
\hline 1113 & Landfill & 1 & 1 & High & 20 & 85 & {$[33.2,42.3]$} & {$[118.2,127.3]$} \\
\hline 1121 & Landfill & 1 & 2 & Low & 20 & 95 & 0 & 95 \\
\hline 1122 & Landfill & 1 & 2 & Medium & 60 & 95 & {$[4.0,20.5]$} & {$[99.0,115.5]$} \\
\hline 1123 & Landfill & 1 & 2 & High & 20 & 95 & {$[32.4,47.5]$} & {$[127.4,142.5]$} \\
\hline 1131 & Landfill & 1 & 3 & Low & 20 & 105 & 0 & 105 \\
\hline 1132 & Landfill & 1 & 3 & Medium & 60 & 105 & {$[8.7,21.0]$} & {$[113.7,126.0]$} \\
\hline 1133 & Landfill & 1 & 3 & High & 20 & 105 & {$[41.6,51.7]$} & {$[146.6,156.7]$} \\
\hline 1211 & Landfill & 2 & 1 & Low & 15 & 95 & 0 & 95 \\
\hline 1212 & Landfill & 2 & 1 & Medium & 40 & 95 & {$[0.0,15.5]$} & {$[95.0,110.5]$} \\
\hline 1213 & Landfill & 2 & 1 & Medium-high & 30 & 95 & {$[49.0,71.6]$} & {$[144.0,166.6]$} \\
\hline 1214 & Landfill & 2 & 1 & High & 15 & 95 & {$[81.7,100.8]$} & {$[176.7,195.8]$} \\
\hline 1221 & Landfill & 2 & 2 & Low & 15 & 105 & 0 & 105 \\
\hline 1222 & Landfill & 2 & 2 & Medium & 40 & 105 & {$[0.0,15.5]$} & {$[105.0,120.5]$} \\
\hline 1223 & Landfill & 2 & 2 & Medium-high & 30 & 105 & {$[53.7,66.6]$} & {$[158.7,171.6]$} \\
\hline 1224 & Landfill & 2 & 2 & High & 15 & 105 & {$[88.3,100.3]$} & {$[193.3,205.3]$} \\
\hline 1231 & Landfill & 2 & 3 & Low & 15 & 115 & 0 & 115 \\
\hline 1232 & Landfill & 2 & 3 & Medium & 40 & 115 & {$[4.0,20.5]$} & {$[119.0,135.5]$} \\
\hline 1233 & Landfill & 2 & 3 & Medium-high & 30 & 115 & {$[58.7,71.6]$} & {$[173.7,186.6]$} \\
\hline 1234 & Landfill & 2 & 3 & High & 15 & 115 & {$[56.1,75.2]$} & {$[171.1,190.2]$} \\
\hline 2111 & Incinerator & 1 & 1 & Low & 20 & 55 & 0 & 55 \\
\hline 2112 & Incinerator & 1 & 1 & Medium & 60 & 55 & 0 & 55 \\
\hline 2113 & Incinerator & 1 & 1 & High & 20 & 55 & {$[16.2,33.2]$} & {$[71.2,88.2]$} \\
\hline 2121 & Incinerator & 1 & 2 & Low & 20 & 60 & 0 & 60 \\
\hline 2122 & Incinerator & 1 & 2 & Medium & 60 & 60 & 0 & 60 \\
\hline 2123 & Incinerator & 1 & 2 & High & 20 & 60 & {$[21.6,29.1]$} & {$[81.6,89.1]$} \\
\hline 2131 & Incinerator & 1 & 3 & Low & 20 & 65 & 0 & 65 \\
\hline 2132 & Incinerator & 1 & 3 & Medium & 60 & 65 & 0 & 65 \\
\hline 2211 & Incinerator & 2 & 1 & Low & 15 & 70 & 0 & 70 \\
\hline 2212 & Incinerator & 2 & 1 & Medium & 40 & 70 & 0 & 70 \\
\hline 2213 & Incinerator & 2 & 1 & Medium-high & 30 & 70 & 0 & 70 \\
\hline 2214 & Incinerator & 2 & 1 & High & 15 & 70 & 17.6 & 87.6 \\
\hline 2221 & Incinerator & 2 & 2 & Low & 15 & 80 & 0 & 80 \\
\hline 2222 & Incinerator & 2 & 2 & Medium & 40 & 80 & 0 & 80 \\
\hline 2223 & Incinerator & 2 & 2 & Medium-high & 30 & 80 & 0 & 80 \\
\hline 2224 & Incinerator & 2 & 2 & High & 15 & 80 & {$[11.1,18.1]$} & {$[91.1,98.1]$} \\
\hline 2231 & Incinerator & 2 & 3 & Low & 15 & 90 & 0 & 90 \\
\hline 2232 & Incinerator & 2 & 3 & Medium & 40 & 90 & 0 & 90 \\
\hline 2233 & Incinerator & 2 & 3 & Medium-high & 30 & 90 & 0 & 90 \\
\hline 2234 & Incinerator & 2 & 3 & High & 15 & 90 & 48.3 & 138.3 \\
\hline \multicolumn{3}{|c|}{ Satisfaction degree: } & \multicolumn{6}{|c|}{$\lambda_{\mathrm{opt}}^{ \pm}=[0.21,0.93]$} \\
\hline \multicolumn{3}{|c|}{ Net system cost $\left(\$ 10^{6}\right)$ : } & \multicolumn{6}{|c|}{$f_{\mathrm{opt}}^{ \pm}=[115.8,191.1]$} \\
\hline
\end{tabular}

conditions where a higher waste generation rate (and thus higher system cost may exist. The $\lambda^{ \pm}$level represents the possibility of satisfying both objective and constraints. It corresponds to the decision makers' preference regarding environmental and economic tradeoffs. In detail, $\lambda^{-}=0.21$ corresponds to the upper-bound system cost ( $f^{+}=\$ 191.1$ million), representing the maximum degree of satisfaction under demanding system conditions. In comparison, $\lambda^{+}=0.93$ is associated with the lower-bound system cost $\left(f^{-}=\$ 115.8\right.$ million), representing the maximum degree of satisfaction under advantageous conditions.

\section{Conclusions}

In this study, an interval-fuzzy two-stage linear programming (IFTP) method has been developed for planning wastemanagement systems under uncertainty. Methods of two-stage stochastic programming and interval fuzzy linear programming are introduced to a general optimization framework to effectively tackle uncertainties that are presented in terms of 
probability density functions, fuzzy membership functions and discrete intervals. Moreover, the IFTP can be used for examining various policy scenarios that are associated with different levels of economic penalties when the promised targets are violated. Furthermore, the IFTP can produce solutions for not only the decision variables and the objective function but also the degree of overall system satisfaction under various conditions. Thus, the solutions can help quantify relationships between the system cost and the overall satisfaction degree, which is meaningful for supporting more in-depth analyses of tradeoffs between environmental and economic objectives as well as those between system optimality and reliability. The method has been applied to a case study of waste-flow allocation within a municipal solid waste management system. The results indicate that reasonable solutions have been generated. They can provide desired waste-flow patterns with minimized system cost and maximized system reliability. In general, although the developed method is for the first time introduced to the waste-management field, the results suggest that it is also applicable to other resource and environmental management problems.

Acknowledgments. This research has been supported by the Major State Basic Research Development Program of China (2005CB72 4201, 2005CB724207, and 2006CB403307) and the Natural Science and Engineering Research Council of Canada. The authors are grateful to the editors and the anonymous reviewers for their insightful comments and suggestions.

\section{References}

Baetz, B.W. (1990). Capacity planning for waste management systems, Civ. Eng. Syst., 7, 229-235.

Birge, J.R. and Louveaux, F.V. (1988). A multicut algorithm for twostage stochastic linear programs, European Journal of Operational Research, 34, 384-392.

Birge, J.R. and Louveaux, F.V. (1997). Introduction to Stochastic Programming, Springer, New York, NY.

Chanas, S. and Zielinski, P. (2000). On the equivalence of two optimization methods for fuzzy linear programming problems, European Journal of Operational Research, 121, 56-63, doi:10.1016/S0 377-2217(99)00011-9.

Chang, N.B., Chen, Y.L. and Wang, S.F. (1997). A fuzzy interval multiobjective mixed integer programming approach for the optimal planning of solid waste management systems, Fuzzy Sets Systems, 89, 35-59, doi:10.1016/S0165-0114(96)00086-3.

Chang, N.B. and Wang, S.F. (1997). A fuzzy goal programming approach for the optimal planning of metropolitan solid waste management systems, Journal of Operation Research, doi:32, 303-321 10.1016/S0377-2217(96)00024-0.

Dantzig, G.B. (1983). Reminiscences about the origins of linear programming, in Bachem, Grostschel and Korte (eds.), Mathematical Programming: The State of the Art, Springer Verlag, Berlin.

Ferrero, R.W., Rivera, J.F. and Shahidehpour, S.M. (1998). A dynamic programming two-stage algorithm for long-term hydrothermal scheduling of multireservoir systems, IEEE-Transactions on Power Systems, 13, 1534-1540, doi:10.1109/ 59.736302.

Huang, G.H., Baetz, B.W. and Patry, G.G. (1992). An interval linear programming approach for municipal solid waste management planning under uncertainty, Civ. Eng. Syst., 9, 319-335.

Huang, G.H., Baetz, B.W. and Patry, G.G. (1993). A grey fuzzy linear programming approach for municipal solid waste management planning under uncertainty, Civ. Eng. Syst., 10, 123-146.

Huang, G.H. and Loucks D.P. (2000). An inexact two-stage stochastic programming model for water resources management under uncertainty, Civ. Eng. Environ. Syst., 17, 95-118, doi:10.1080/ 0263025 0008970277.

Huang G.H., Sae-Lim, N., Liu, L. and Chen, Z. (2001). An intervalparameter fuzzy-stochastic programming approach for municipal solid waste management and planning, Environ. Model. Assess., 6, 271-283.

Li, Y.P. and Huang, G.H. (2006a). Minimax regret analysis for municipal solid waste management: An interval-stochastic programming approach, J. Air Waste Manage. Assoc., 56, 931-944.

Li, Y.P. and Huang, G.H. (2006b). An inexact two-stage mixed integer linear programming method for solid waste management in the City of Regina, Journal of Environmental Management, 81, 188209, doi:10.1016/j.jenvman.2005.10.007.

Li, Y.P., Huang, G.H., Nie, S.L., Nie, X.H. and Maqsood, I. (2006). An interval-parameter two-stage stochastic integer programming model for environmental systems planning under uncertainty, Eng. Optimiz., 38(4), 461-483 , doi:10.1080/03052150600557742.

Li, Y.P., Huang, G.H., Nie, S.L. and Qin, X.S. (2007). ITCLP: An inexact two-stage chance-constrained program for planning waste management systems, Resour. Conserv. Recycling, 49(3), 284-307, doi:10.1016/j.resconrec.2006.03.017.

Loucks, D.P., Stedinger, J.R., and Haith, D.A. (1981). Water Resource Systems Planning and Analysis, Prentice-Hall, Englewood Cliffs, N.J.

Luo, B., Maqsood, I., Yin, Y.Y., Huang, G.H. and Cohen, S.J. (2003). Adaption to climate change through water trading under uncertainty - an inexact two-stage nonlinear programming approach, J. Env. Inform., 2(2), 58-68, doi:10.3808/ jei.2003 00022.

Maqsood, I. and Huang, G.H. (2003). A two-stage interval-stochastic programming model for waste management under uncertainty, J. Air Waste Manage. Assoc., 53(5), 540-552.

Ruszczynski, A. and Swietanowski, A. (1997). Accelerating the regularized decomposition method for two-stage stochastic linear problems; European Journal of Operational Research, 101, 328-342, doi:10.1016/S0377-2217(96)00401-8.

Schultz, R., Stougie, L. and Vander. Vlerk, M.H. (1996). Two-stage stochastic integer programming: a survey, Statistical Neerlandica, 50(3), 404-416.

Seifi, A., and Hipel, K. W. (2001). Interior-point method for reservoir operation with stochastic inflows, ASCE-J. Water Resour. Plng. and Mgmt., 127(1), 48-57, doi:10.1061/(ASCE)0733-9496 (2001) 127:1(48).

Yeomans, J.S. and Huang, G.H. (2003). An evolutionary grey, hop, skip, and jump approach: generating alternative policies for the expansion of waste management, J. Env. Inform., 1(1), 37-51, doi:10. 3808/jei.200300005.

Yeomans, J.S., Huang, G.H., and Yoogalingam, R. (2003). Combining simulation with evolutionary algorithms for optimal planning under uncertainty: an application to municipal solid waste management planning in the Reginonal Municipality of Hamilton-Went worth, J. Env. Inform., 2(1), 11-30, doi:10.3808 /jei.200300014.

Zimmermann, H.J. (1985). Fuzzy Set Theory and its Applications, Kluwer-Nijhoff Publishing. 